\title{
The importance of serum interleukin-20 levels in patients with Behçet's disease
}

\author{
Mehmet Emin Enecik ${ }^{1, A-E}$, Barış Mavii, ${ }^{2, A-C, E}$, Çiğdem Yücele, ${ }^{3, C-E}$, Göksal Keskin ${ }^{4, A, E, F}$, Mehmet Yıldız ${ }^{5, B, C}$ \\ ${ }^{1}$ Department of Rheumatology, Ankara Numune Training and Research Hospital, Turkey \\ ${ }^{2}$ Department of Internal Medicine, Mersin State Hospital, Turkey \\ ${ }^{3}$ Department of Clinical Biochemistry, Ankara Numune Training and Research Hospital, Turkey \\ ${ }^{4}$ Department of Immunology and Allergy, Medical Faculty, Ankara University, Turkey \\ ${ }^{5}$ Department of Internal Medicine, Dışkapı Yıldırım Beyazıt Training and Research Hospital, Ankara, Turkey \\ A - research concept and design; $\mathrm{B}$ - collection and/or assembly of data; $\mathrm{C}$ - data analysis and interpretation; \\ $D$ - writing the article; $E$ - critical revision of the article; $F$ - final approval of the article
}

\section{Address for correspondence}

Çiğdem Yücel

E-mail: yucelcigdem80@gmail.com

\section{Funding sources}

None declared

\section{Conflict of interest}

None declared

Received on November 24, 2016

Reviewed on January 14,2017

Accepted on April 19, 2017

\section{Abstract}

Background. Behçet's disease (BD) is a complex multisystemic disease with an unknown origin, which presents with aphthous and genital ulcers, cutaneous lesions, arthritis, ocular lesions, and defects in the gastrointestinal and central nervous systems.

Objectives. In this study, we examined the relationship between serum interleukin-20 (IL-20) levels and disease activity in BD patients.

Material and methods. A total of 45 BD patients diagnosed according to the BD diagnosis criteria determined by the International Study Group for Behçet's Disease were included in the study. Out of 45 patients, 17 had inactive BD and 28 had active BD. The control group consisted of 25 healthy subjects. The IL-20 levels of all the groups were detected and compared with each other. Serum IL-20, erythrocyte sedimentation rate (ESR) and (-reactive protein (CRP) levels were examined.

Results. The IL-20 levels of the active BD patient group were significantly higher than in the control group $(p<0.001)$ and in the inactive BD patient group $(p<0.001)$. No statistically significant difference was detected between the lL-20 levels of the control group and the inactive BD patient group ( $p=0.2)$.

Conclusions. Higher IL-20 levels in active BD patients, when compared to inactive BD patients and healthy controls indicate that the disease is an inflammatory one and IL-20 plays a role in the disease pathogenesis. Moreover, it can be concluded that IL-20 might have a role in the complex process of the settlement and activation of the disease.

Key words: cytokines, inflammation, Behçet's disease, interleukin-20

DOI

10.17219/acem/70523

\section{Copyright}

Copyright by Author(s)

This is an article distributed under the terms of the

Creative Commons Attribution Non-Commercial License

(http://creativecommons.org/licenses/by-nc-nd/4.0/) 


\section{Introduction}

Behçet's disease (BD) is a chronic, recurrent inflammatory disease capable of affecting multiple systems; it is mostly presented with mucocutaneous, ocular, articular, vascular, and neurological symptoms. Although the etiology of the disease is not yet fully understood, it has been related to viral, bacterial, genetic, environmental, psychological basis; toxic, coagulative and immune factors have also been suspected., ${ }^{1,2}$ Neutrophil hyperfunction, vasculitis and autoimmune response are the 3 major pathophysiological changes that play an important role in the disease pathogenesis. ${ }^{3}$ After the publication of studies which showed an increase in immunoglobulins, immune complexes, complement, and acute phase reactants in $\mathrm{BD}$, the disease was classified among autoimmune disorders. ${ }^{4}$ Cytokines predominate in most immune mechanisms. These proteins are synthesized from several cell types which are important mediators in different immune mechanisms. Proinflammatory cytokines and mediators can be effective in BD prognosis. ${ }^{5}$ Many studies have been carried out on serum cytokine levels to enlighten the etiopathogenesis of $\mathrm{BD}$. These include interleukins (IL): IL-2, IL-4, IL-6, IL-8, IL-10, IL-12, IL-17, IL-18, and also interferon gamma (IFN- $\gamma$ ) and tumor necrosis factor alpha (TNF- $\alpha){ }^{1,4-7}$

Interleukin-20 (IL-20) is a cytokine that belongs to the IL-10 family with an amino acid sequence very similar to that of IL-10. It is expressed from the active monocytes, keratinocytes and endothelial cells. ${ }^{8}$ Interleukin-20 exerts its biological effects via type I and type II receptors composed of IL-20 R1 and IL-20 R2 complex chains, respectively. Intracellular domains of both receptors are in contact with Janus kinase 1 (Jak1) and tyrosine kinase 2 (Tyk2). ${ }^{9,10}$

The importance of IL-20 receptors has been shown in several inflammatory diseases, but the mechanism of action and pathophysiological significance is not yet fully understood. T cells and cytokines play an important role in immunological disorders. A variety of proinflammatory cytokines are expressed, including IL-1, IL-2, IL-6, IL-8, IL-12, IFN- $\gamma$, and TNF- $\alpha$. Among these proinflammatory cytokines, IL-1 $\beta$ and TNF- $\alpha$ have proven to induce IL-20 secretion, especially from cells like macrophages and synoviocytes. Interleukin-20 itself increases the contribution of neutrophiles, monocytes and $\mathrm{T}$ cells to inflammation by directly inducing monocyte chemoattractant protein 1 (MCP-1) and IL-8 production. Several studies have demonstrated that IL-20 plays a role in inflammatory diseases, such as rheumatoid arthritis, psoriasis and atherosclerosis. ${ }^{11-13}$

In the study on atherosclerotic plaques, IL-20 was shown to increase the synthesis of fibroblast growth factor ( $\beta$-FGF), vascular endothelial cell growth factor (VEGF) and matrix metalloproteinase-2 (MMP-2), which are related to chronic inflammation and atherosclerotic angiogenesis. In the study conducted in the patients with rheumatoid arthritis, IL-20 has been shown to increase the synthesis of TNF- $\alpha$, IL-1 $\beta$, IL- 6 , IL- 8 , and MCP- $1 .{ }^{14}$

In this study, we concentrated on serum IL-20 levels. We compared serum IL-20 levels with levels of erythrocyte sedimentation rate (ESR) and C-reactive protein (CRP), which are known to be elevated in inflammation. We aimed to detect the role of IL-20 in BD pathogenesis and its relation with disease activity.

\section{Material and methods}

A total of 45 patients (20 male, 25 female) diagnosed with $\mathrm{BD}$ according to the diagnosis criteria determined by the International Study Group for Behçet's Disease, admitted to the Clinic of Immunology in the Dişkapı Yıldırım Beyazıt Training and Research Hospital (Ankara, Turkey) were included in the study (17 inactive and 28 active patients). Patients having any other acute or chronic inflammatory disease which might affect IL-20 levels were excluded from the study. The control group was composed of 25 healthy individuals ( 16 male, 9 female) with no acute or chronic illness. Behçet's disease activity was determined by patients filling out the Behçet's Disease Current Activity Form (BDCAF). ${ }^{15,16}$

Study groups were designed as BD patients (active and inactive groups) and healthy controls. Demographic features of all participants were recorded. Venous blood samples were collected from the participants after $12 \mathrm{~h}$ of fasting. Blood samples collected for IL-20 analysis were centrifuged at $4000 \mathrm{rpm}$ for $10 \mathrm{~min}$. Separated sera were aliquoted into Eppendorf tubes and stored at $-80^{\circ} \mathrm{C}$ until the time of analysis. USCN Human IL-20 ELISA kit (USCN Life Science Inc., Wuhan, China) was used for serum IL-20 level detection. Prior to the study, the frozen specimens were allowed to melt at room temperature. Apart from IL-20 levels, also CRP, ESR and white blood cell (WBC) parameters, which are known to be related with disease activity, were detected from the same samples. C-reactive protein was studied with the immunoturbidimetric method, while ESR was studied with the Westergren method, and WBC analysis was made by the volume, conductivity, scatter (VCS) technique.

\section{Ethical approval}

All procedures performed in studies involving human participants were in accordance with the ethical standards of the institutional and/or national research committee and with the 1964 Helsinki declaration and its later amendments or comparable ethical standards. The study was approved by the ethical committee (No. 02.04.2010/16).

Informed consent was obtained from all individual participants included in the study. 


\section{Statistical analysis}

Data analysis was made with Statistical Package for the Social Sciences (SPSS) for Windows v. 11.5 software (SPSS Inc., Chicago, USA). The normality tests were made with the Shapiro-Wilk test. Descriptive statistics of normally distributed continuous variables are illustrated as mean with standard deviation (SD). Descriptive statistics of nonnormally distributed continuous variables are illustrated as median (interquartile range) values. Differences of normally distributed variables were analyzed with Student's t-test, while non-normally distributed variables were analyzed with the Mann-Whitney U test. The Kruskal-Wallis test was used for more than 2 groups. The nonparametric multiple comparison test was used when the KruskalWallis test results appeared to be significant.

Nominal variables were analyzed with Pearson's $\chi^{2}$ test. A correlation between continuous variables was detected with Spearman's correlation test. Probability value $\mathrm{p}<0.05$ was accepted as statistically significant for all tests.

\section{Results}

The patient group consisted of 45 patients, 20 males (44\%) and 25 females (55.6\%), while the control group consisted of 16 males (64\%) and 9 females (36\%). The mean age of the patient and control groups was $41.5 \pm 11$ years and $29.2 \pm 5.3$ years, respectively (Table 1 ). Among the patients diagnosed with BD, 18 (40\%) had aphthous ulcers, 9 (20\%) had genital ulcers, 6 (13.3\%) had uveitis, 5 (11.1\%) had acneiform skin lesions and erythema nodosum, 3 (6.7\%) had neurological findings, 2 (4.4\%) had pulmonary thromboembolism, and 9 (20\%) had arthralgia (Table 2). When compared with the control group, the IL-20 levels of the active patient group were significantly higher $(\mathrm{p}<0.001)$. Moreover, the IL-20 levels of the active patient group were higher than the IL-20 levels of the inactive patient group, and the difference was statistically significant $(\mathrm{p}<0.001)$. There was no statistically significant difference between the IL-20 levels of the inactive patients and the control group $(\mathrm{p}=0.2)$ Erythrocyte sedimentation rate was higher in the patient group $(\mathrm{p}<0.001)$ (Table 3$)$. Also, serum CRP levels were significantly higher in the patient group when compared to the control group ( $\mathrm{p}<0.001)$ (Table 3$)$.

In symptomatic $\mathrm{BD}$ patients, a moderate positive correlation between serum IL-20 levels and symptoms was

Table 1. Demographical features of BD group and control group

\begin{tabular}{|l|c|c|}
\hline \multicolumn{1}{|c|}{ Feature } & Control group & BD group \\
\hline Age [years] & $29.2 \pm 5.3$ & $41.5 \pm 11.0$ \\
\hline $\begin{array}{l}\text { Gender, } \mathrm{n}(\%) \\
\text { male } \\
\text { female }\end{array}$ & $16(64)$ & $20(44.4)$ \\
\hline
\end{tabular}

$\mathrm{BD}$ - Behçet's disease.
Table 2. Distribution of clinical findings among active BD patients $(n=28)$

\begin{tabular}{|l|c|}
\multicolumn{1}{|c|}{ Clinical findings } & Number of patients (\%) \\
\hline Aphthous ulcers & $18(40.0)$ \\
\hline Genital ulcers & $9(20.0)$ \\
\hline Uveitis & $6(13.3)$ \\
\hline Skin lesions & $5(11.1)$ \\
\hline Neurological findings & $3(6.7)$ \\
\hline Pulmonary thromboembolism & $2(4.4)$ \\
\hline Arthralgia & $9(20.0)$ \\
\hline
\end{tabular}

$\mathrm{BD}$ - Behçet's disease.

Table 3. Laboratory parameters of the study groups

\begin{tabular}{|l|c|c|c|}
\hline \multicolumn{1}{|c|}{ Parameter } & Control group & BD group & p-value \\
\hline IL-20 [pg/mL] & $16.5(0.0-140.8)$ & $133.4(0.0-1000.0)$ & $<0.001$ \\
\hline ESR $[\mathrm{mm} / \mathrm{h}]$ & $8.0(1.0-11.0)$ & $22.0(1.0-100.0)$ & $<0.001$ \\
\hline CRP $[\mathrm{mg} / \mathrm{L}]$ & $3.2(3.2-3.2)$ & $3.4(3.2-108.0)$ & $<0.001$ \\
\hline
\end{tabular}

BD - Behçet's disease, IL-20 - interleukin-20; ESR - erythrocyte sedimentation rate; CRP - C-reactive protein.

detected $(\mathrm{r}=0.604 ; \mathrm{p}<0.001)$. No significant correlation was found between serum IL-20 levels and ESR and CRP parameters $(\mathrm{r}=0.268 ; \mathrm{p}=0.075$ and $\mathrm{r}=0.001 ; \mathrm{p}=0.995$, respectively) (Table 4 ).

The IL-20 levels in patients with aphthous ulcers were significantly higher than the in patients without aphthous ulcers $(\mathrm{p}=0.006)$ (Table 5). The IL-20 levels of patients with or without genital ulcers, uveitis, skin lesions, and arthralgia did not show any significant difference ( $\mathrm{p}$-values: $0.232,0.181,0.207$, and 0.181 , respectively).

Table 4. ESR, CRP levels and clinical findings within BD group, their correlations with serum IL-20 levels and significance levels

\begin{tabular}{|l|c|c|}
\hline \multicolumn{1}{|c|}{ Variable } & Correlation coefficient & p-value \\
\hline ESR & 0.268 & 0.075 \\
\hline CRP & 0.001 & 0.995 \\
\hline Number of clinical findings & 0.604 & $<0.001$ \\
\hline
\end{tabular}

ESR - erythrocyte sedimentation rate; CRP - C-reactive protein; $\mathrm{BD}$ - Behçet's disease.

Table 5. Serum IL-20 levels of patients with and without aphthous ulcers

\begin{tabular}{|c|c|c|c|}
\hline Variable & Aphthous ulcers (+) & Aphthous ulcers (-) & p-value \\
\hline $\mathrm{IL}-20[\mathrm{pg} / \mathrm{mL}]$ & $223.7(82.9-1000.0)$ & $90.8(0.0-608.0)$ & 0.006 \\
\hline
\end{tabular}

IL-20 - interleukin-20.

\section{Discussion}

Behçet's disease is a chronic, recurrent inflammatory disease which is presented with mucocutaneous, ocular, articular, vascular, and neurological symptoms. Neutrophil hyperfunction, vasculitis and autoimmune response are 
the 3 major pathophysiological changes that play an important role in the disease pathogenesis. Although the etiology of the disease is not yet fully understood, it has been related to viral, bacterial, genetic, environmental, psychological, toxic, and immune factors. The most common hypothesis is that in patients with a genetic tendency to the disease, an uncontrolled immune response occurs, which in turn is triggered by infective (viral, bacterial, etc.) agents, heat shock proteins or autoantigens, and results in the occurrence of vasculitis. ${ }^{4}$

After the publication of studies which showed increases in immunoglobulins, immune complexes, complement, and acute phase reactants in BD, this disease was classified among autoimmune disorders. ${ }^{5,6}$ Cytokines are known to be predominant in several immune mechanisms. These proteins are synthesized in different cell types which are important in immune-inflammatory reactions. Proinflammatory cytokines may also have a role in $\mathrm{BD}$ prognosis. ${ }^{13}$

To enlighten the etiopathogenesis of $\mathrm{BD}$, several studies on serum cytokines, including IL-2, IL-4, IL-6, IL-8, IL-10, IL-12, IL-17, IL-18, IFN- $\gamma$, TNF- $\alpha$, and leptin, have been carried out. ${ }^{7}$ Interleukin-20 is a member of the IL10 family of cytokines. It is expressed from the monocytes, keratinocytes, synoviocytes, and endothelial cells. ${ }^{8}$ The family of IL-10 cytokines are a group of cytokines encoded in the same genomic region, showing similarities in protein structures and in the receptors they use. Interleukin-20 exerts its effects over type-1 and type- 2 receptors. These receptors have shown to be expressed from 25 different tissues, including the brain, kidneys, heart, lungs, small intestine, and skin. ${ }^{17}$

In many inflammatory diseases, the importance of IL-20 and its receptors have been proven. But the mechanism of action and pathophysiological significance remains uncertain. Cytokines and T cells play an important role in disorders with immunological basis. Several proinflammatory cytokines are exposed, including IL-1, IL-2, IL-6, IL-8, IL-12, IFN- $\gamma$, and TNF- $\alpha$. Among these cytokines, IL-1 $\beta$ and TNF- $\alpha$ are known to induce IL-20, especially from cells like macrophages and synoviocytes. Indeed, IL-20 is known to induce the activity of neutrophiles, monocytes and $\mathrm{T}$ cells in inflammation by increasing MCP-1 and IL- 8 expression. ${ }^{11-13}$ Inflammatory cytokines like TNF- $\alpha$, IL-6 and IL- 8 have been shown to increase in BD and correlate positively with the disease activity. ${ }^{8}$ That is why IL-20 may also play a role in the pathogenesis of $\mathrm{BD}$. When the skin samples of psoriasis patients and healthy controls were compared, IL-20 expression was shown to be elevated clearly in the areas of skin lesions; in another study with polymerase chain reaction (PCR), IL-20 levels were found to be 100 times higher than IFN- $\gamma$ levels in the psoriatic skin. ${ }^{12,17-20}$ In a study conducted in 2004, IL-20-IL-20 receptor complex expression was shown in both the synovial membranes and synovial fibroblasts of patients with rheumatoid arthritis. That study demonstrated that IL-20 induces the expression of proinflammatory cytokines, like TNF- $\alpha$ and IL-1, in the synovial fibroblasts, which indeed plays an important role in the pathogenesis of rheumatoid arthritis. ${ }^{21}$ In 2008, Li et al. claimed that IL-22, IL-19 and IL-20 played a role in the pathogenesis of autoimmune uveitis and autoimmune intraocular inflammatory disease with increased gene expressions. The study was performed on the RNA samples of 50 patients with noninfectious autoimmune uveitis. ${ }^{22}$

In this study, we compared the IL-20 serum levels of BD patients with those of healthy controls. Besides this, we compared IL-20 levels in active and inactive disease states to verify if IL-20 can be an activation marker, like CRP, ESR and WBC parameters. Serum IL-20 levels were significantly higher in the patient group when compared to the control group. Especially in the active BD patients, serum IL-20 levels were significantly higher than in the control group. The reasons underlying this situation might be that IL-20 is a proinflammatory cytokine and it contributes to the settlement and maintenance of chronic inflammation, and many other unknown factors. There was no statistically significant difference between the serum IL-20 levels of the control and inactive patient groups. This may be due to suppressed immunological reaction and inflammation in the inactive patient group under the influence of medication. Besides this, CRP, ESR and WBC levels were detected to be higher in the patient group when compared to the control group.

\section{Conclusions}

Higher IL-20 serum levels in BD patients in comparison to the control group and were found. Also, higher IL-20 levels in the active group, when compared to the inactive group, and the correlation of IL-20 levels with standard activation markers, like CRP and ESR, present the possibility that IL-20 plays a role in the complex process of disease formation and activation. However, more detailed and large population-based studies need to be carried out to confirm the direct role of IL-20 in BD pathogenesis.

\section{References}

1. Mat MC, Sevim A, Fresko I, Tüzün Y. Behçet's disease as a systemic disease. Clin Dermatol. 2014;32:435-442.

2. Yurdakul S, Hamuryudan V, Yazıcı H. Behçet syndrome. Curr Opin Rheumatol. 2004;16:38-42.

3. Banfioli AA, Orefice F. Behçets disease. Semin Ophthalmol. 2005;20(3):199-206.

4. Akdeniz N, Esrefoglu M, Keleş MS, Karakuzu A, Atasoy M. Serum interleukin-2, interleukin-6, tumour necrosis factor-alpha and nitric oxide levels in patients with Behcet's disease. Ann Acad Med Singapore. 2004;33:596-599.

5. Hamzaoui K, Hamzaoui A, Guemira F, Bessioud M, Hamza M, Ayed K. Cytokine profile in Behçet's disease patients. Relationship with disease activity. Scand J Rheumatol. 2002;31:205-210.

6. Adam B, Calikoglu E. Serum interleukin-6, procalcitonin and C reactive protein levels in subjects with active Behçet's disease. J Eur Acad Dermatol Venereol. 2004;18:318-320. 
7. Aridogan BC, Yildirim M, Baysal V, Inaloz HS, Baz K, Kaya S. Serum levels of IL-4, IL-10, IL-12, IL-13 and IFN-gamma in Behçet's disease. J Dermatol. 2003;30:602-607.

8. Volk H, Asadullah K, Gallagher G, Sabat R, Grutz G. IL-10 and its homologs: Important immune mediators and emerging immunotherapeutic targets. Trend Immunol. 2001;22(8):414-417.

9. Blumberg $\mathrm{H}$, Conklin D, Xu WF, et al. Interleukin 20: Discovery, receptor identification, and role in the epidermal function. Cell. 2001;104(1):9-19.

10. Dumoutier L, Leemans C, Lejeune D, Kotenko SV, Renauld JC. Cutting edge: STAT activation by IL-19, IL-20 and mda-7 through IL-20 receptor complexes of two types. JImmunol. 2001;167(7):3545-3549.

11. Kragstrup TW, Otjaker K, Holm C, et al. The expression of IL-20 and IL-24 and their shared receptors are increased in rheumatoid arthritis and spondyloarthropathy. Cytokine. 2008;41:16-23.

12. Wei CC, Chen WY, Wang YC, et al. Detection of IL-20 and its receptors on psoriatic skin. Clin Immunol. 2005;117(1):65-72.

13. Hsieh MY, Chen WY, Jiang MJ, et al. IL-20 promotes angiogenesis in a direct and indirect manner. Genes Immun. 2006;7(3):234-242.

14. Panutsopulos D, Papalambros E, Sigala F, Zafiropoulos A, Arvantis DL, Spandidos DA. Protein and mRNA expression levels of VEGF-A and TGF-beta1 in different types of human coronary atherosclerotic lesions. Int J Mol Med. 2005;15(4):603-610.

15. Bodur H, Borman P, Ozdemir Y, Atan C, Kural G. Quality of life and life satisfaction in patients with Behçet's disease: Relationship with disease activity. Clin Rheumatol. 2006;25:329-333.

16. Lawton G, Chamberlain MA, Bhakta BB, Tennant A. Adv Exp Med Biol. 2003;528:149-151.

17. Kunz S, Wolk K, Whitte, et al. Interleukin-19, IL-20 and IL-24 are produced by and act on keratinocytes and are distinct from classical ILs. Exp Dermatol. 2006;15(12):991-1004.

18. Li HH, Lin YC, Chen PJ, et al. Interleukin-19 upregulates keratinocyte growth factor and is associated with psoriasis. $\mathrm{Br} J$ Dermatol. 2005;153(3):591-595.

19. Otkjaer K, Kragballe K, Funding AT, et al. The dynamics of gene expreesion of IL-19 and IL-20 and their receptors in psoriasis. Br J Dermatol. 2005;153(5):911-918.

20. Romer J, Hasselager E, Norby PL, et al. Epidermal overexpression of interleukin-19 and -20 mRNA in psoriatic skin disappears after short term treatment with cyclosporine a or calcipotriol. J Invest Dermatol. 2003;121(6):1306-1311.

21. Smeets TJM, Chandrasekher Y, Haringman JJ, Tak PP. IL-20 is expressed in inflamed synovium of patients with psoriatic arthritis and rheumatoid arthritis. Arthritis Res Therapy. 2004;6:6-9.

22. Li Z, Liu B, Arvydase M, et al. Gene expression profiling in autoimmune non-infectious uveitis disease. J Immunol. 2008;181(7):5147-5157. 